\title{
Interferon- $\gamma$ release assays for tuberculous meningitis diagnosis: a meta-analysis
}

\author{
You Lan, Wei Chen, Qun Yan, Wenen Liu
}

Department of Clinical Laboratory, Xiangya Hospital Central South University,
Changsha, Hunan, China

Submitted: 21 March 2018; Accepted: 22 August 2018

Online publication: 3 March 2020

Arch Med Sci 2021; 17 (5): 1241-1250

DOI: https://doi.org/10.5114/aoms.2019.86994

Copyright $\odot 2019$ Termedia \& Banach

\section{Abstract}

Introduction: Tuberculous meningitis (TBM) is still a great challenge to global public health. As conventional diagnostic methods for TBM are unsatisfactory, interferon- $\gamma$ release assays (IGRAs) have been introduced for TBM diagnosis tentatively. However, the role of IGRAs for diagnosing TBM remains unclear. Thus, we systematically evaluated the diagnostic performance of cerebrospinal fluid (CSF) and peripheral blood (PB) IGRAs in TBM to fill this blank.

Material and methods: Relevant studies were systematically searched in both foreign and Chinese databases up to March 2018. Studies in which TBM diagnosis was based on microbiological or clinical criteria were included. The quality of the included studies was assessed through the Quality Assessment of Diagnostic Accuracy Studies-2 (QUADAS-2) tool. Main outcome measures, including sensitivity, specificity, positive likelihood ratio (PLR), negative likelihood ratio (NLR) and diagnostic odds ratio (DOR), were pooled statistically using random effects models. The potential heterogeneity was explored by threshold effect analysis, subgroup analyses and meta-regression. Funnel plots and Egger's test were used to test the potential publication bias. Statistical analyses were performed using Stata and Meta-DiSc software.

Results: Twenty-six out of 656 publications were eligible for meta-analysis, including 1892 participants in total. The pooled estimates of PB IGRAs for TBM diagnosis are as follows: sensitivity: $0.81(95 \% \mathrm{Cl}$ : 0.78-0.84); specificity: 0.76 (95\% Cl: 0.73-0.78); PLR: 4.23 (95\% Cl: 2.95-6.07); NLR: 0.24 (95\% Cl: 0.19-0.32) and DOR: 21.06 (11.91-37.24). The corresponding estimates for CSF IGRAs were obtained: sensitivity: 0.81 (95\% Cl: 0.76-0.85); specificity: 0.89 (95\% Cl: 0.86-0.92); PLR: 7.87 (95\% Cl: 4.98-12.46); NLR: 0.19 (95\% Cl: 0.13-0.29); and DOR: 47.74 (25.02-91.12).

Conclusions: The diagnostic performance of IGRAs is suboptimal. In terms of cost, turn-around time and accessibility, these assays are unsuitable for use as biomarkers for TBM diagnosis.

Key words: interferon- $\gamma$ release assays, tuberculous meningitis, diagnosis.

\section{Introduction}

Tuberculous meningitis (TBM) has historically been a serious threat to public health. World Health Organization (WHO) estimated that in 2016 there were 10.4 million people who fell ill with tuberculosis (TB) worldwide and 1.3 million deaths after infection [1]. TBM, the most severe form of extrapulmonary tuberculosis, accounts for $1-2 \%$ of all cases of TB and kills or severely disables approximately one half of infected people [2]. Diagnosis of TBM is always a challenge due to its non-specific clinical

\author{
Corresponding author: \\ Wenen Liu \\ Department \\ of Clinical Laboratory \\ Xiangya Hospital Central \\ South University \\ 87 Xiangya Road \\ Changsha, Hunan (410008) \\ China \\ Phone: +86-0731-84327440 \\ E-mail: wenenliu@163.com
}


presentation. As a result, appropriate treatment is often delayed, which is frequently associated with high mortality and long-term disability for patients [3]. Early diagnosis of TBM is vital for successful disease management. Thus, a rapid diag nostic approach to TBM with improved accuracy compared to existing methods is required.

Conventional methods, such as acid fast bacilli (AFB) on smear and culture of cerebrospinal fluid (CSF), are the absolute criteria to diagnose TBM. These methods are low-cost and widely used in resource-poor settings [2, 4]. Nevertheless, the sensitivity of smear microscopy is impaired by the low number of bacilli present in CSF. Culture is more sensitive but it may take up to 42 days, which often delays clinical decision-making $[5,6]$. Another method, the tuberculin skin test (TST), widely used in developing countries, can be affected by a previous Bacillus Calmette-Guérin (BCG) vaccination and non-tuberculous mycobacteria (NTM) such as Mycobacterium marinum, M. kansasii and M. abscessus [7]. Molecular techniques are employed increasingly in low-income countries on account of their rapid diagnosis. However, the sensitivity of these assays is variable, ranging 0.32-1.00, which makes molecular tests less useful in clinical practice and offers little advantage over smear microscopy [8].

An immunodiagnostic method, namely interferon- $\gamma$ release assays (IGRAs), which measures the release of interferon (IFN) after stimulation by Mycobacterium tuberculosis $(M$. $T b$ ) antigens, has provided an alternative method for TBM diagnosis. Two IGRAs, TSPOT.TB and QuantiFERON-TB Gold In-Tube test (QFT-GIT), are now commercially available. The cost of these tests can be up to ten times higher than conventional methods. But, with the unique advantage of short turn-around time and accessibility, their use has increased substantially in the past decade. Many studies on application of IGRAs have been published [9-13]. Furthermore, the IGRAs have been shown to be a useful diagnostic tool with high specificity up to 0.96 when using CSF of patients with TBM in some studies [14, 15].

To date, whether IGRAs can be used to diagnose TBM is still controversial. A systematic review of 8 publications up to August 2015 concluded that the current evidence does not support the use of IGRAs to diagnose TBM [16]. Since then, a growing number of studies have evaluated the performance of IGRAs in TBM diagnosis, but no updated metaanalysis has been performed. Additionally, the review had a language restriction which may lead to the loss of relevant publications as TB is more prevalent in non-English speaking countries. Herein a meta-analysis with a broader search strategy was conducted to comprehensively assess the overall accuracy of IGRAs for TBM diagnosis.

\section{Material and methods}

\section{Search strategy and study selection}

Published studies were systematically collected by searching PubMed, Embase, Cochrane Library, Chinese Biomedical Literature Database (CBM), China National Knowledge Infrastructure (CNKI), China Science and Technology Journal (VIP) and Wanfang databases. The combination of key words was as follows: (tuberculous meningitis, or tuberculosis, meningeal, or extrapulmonary tuberculosis) and (QuantiFERON, or T-SPOT.TB, or TSPOT, or ELISPOT, or Interferon- $\gamma$ assays, or Interferon- $\gamma$ release assays, or IGRA, or T cell assays, or T cell response). Searching was investigated without restriction on language, as TB is more prevalent in non-English speaking countries. Reference lists were searched manually to further identify additional eligible literature.

Studies meeting the following criteria were included: (1) assessed the performance of IGRAs in TBM; (2) adopted predefined, specific diagnostic criteria of TBM, including microbiological and clinical criteria based on clinical presentation, CSF analysis, radiology and responsiveness to anti-tuberculosis chemotherapy; (3) final diagnosis of TBM was independent of the IGRAs result. Studies were excluded if they: (1) were case reports, comments, animal experiments, literature review or meta-analysis; (2) were not diagnostic tests; (3) lacked appropriate study design (e.g., took healthy people as the control group); (4) had fewer than 10 TBM patients; (5) reported insufficient data that were not able to calculate the sensitivity, specificity, positive likelihood ratio $(P L R)$, and negative likelihood ratio (NLR).

\section{Data extraction and quality assessment}

Two reviewers independently checked all potentially relevant studies. Discrepancies were resolved by discussion with other investigators until a consensus was achieved. Data were collected from each study, including first author, year of publication, country, study design, number of cases, IGRA method, sample, true positive (TP), false-positive (FP), false-negative (FN) and true-negative (TN). For papers that evaluated more than one commercial IGRA or using two types of specimens simultaneously, each of them was considered as two independent studies in this meta-analysis and the data were extracted separately.

The methodological quality of the studies was assessed using the Quality Assessment of Diagnostic Accuracy Studies-2 (QUADAS-2) tool by two independent researchers. All disagreements were resolved by consensus. 
Comparison of diagnostic performance among ADA, new techniques and IGRAs for TBM

The adenosine deaminase assay (ADA) is a traditional test for TBM diagnosis. New techniques such as nested real-time polymerase chain reaction (nRT-PCR), one-tube nested PCR-lateral flow strip test (OTNPCR-LFST) and loop-mediated isothermal amplification (LAMP) have been designed for TBM diagnosis since 2016. The diagnostic accuracy of these techniques was reviewed to obtain a better understanding of the IGRA performance.

\section{Statistical analysis}

Standard methods recommended for metaanalyses of diagnostic test evaluations [17] were used. The data were analyzed using Meta-DiSc software (version 1.4) and Stata 12.0. The following measures of test accuracy were computed for each study: sensitivity, specificity, PLR, NLR and diagnostic odds ratio (DOR). In most circumstances, a PLR greater than 10 and an NLR less than 0.1 provide strong diagnostic evidence to rule in or rule out diagnoses respectively [18]. The DOR describes the odds of positive test results in patients with TBM compared with the odds of positive results in those without the disease. It is calculated as DOR $=$ PLR/NLR [19]. Heterogeneity was assessed by chi-square $\left(\chi^{2}\right)$ and $l^{2}$ statistical tests. $P<0.05$ was considered statistically significant for heterogeneity. For the $I^{2}$ statistic, heterogeneity was defined as low, moderate, and high when $l^{2}$ was more than $25 \%, 50 \%$, and $75 \%$, respectively [20]. In this study, a random-effect model was used to pool estimates. The threshold effect was assessed by the Spearman rank correlation test and considered significant if $p<0.05$. To explore the sources of heterogeneity, subgroup analysis and meta-regression were conducted based on parameters such as IGRA method, TB prevalence, blinding method, sample size and reference standard. $P<0.05$ indicates a contribution to heterogeneity. Potential presence of publication bias was tested using funnel plots and Egger's test. A $p$-value $<0.05$ was considered representative of statistical significance [21].

\section{Results}

\section{General information}

Study identification and selection were outlined in Figure 1, where "not primarily relevant to TBM" means publications focused on extrapulmonary tuberculosis but were irrelevant to TBM or did not provide valid TBM information. In the end, 26 [14, 15, 22-45] out of 656 publications were available for the final analysis. Characteristics of the included publications are presented in Table I.

According to the lists of 30 high TB burden countries from WHO Global tuberculosis report 2017 [1], studies conducted in the countries included in the list were defined as high TB burden settings. Apart from three studies $[15,25,32]$ carried out in Korea, most of the studies were conducted in countries with high TB prevalence, such as India, China and Africa. Laboratory investigators were blinded to the clinical data and clinicians were blinded to the laboratory results in 7 publications $[14,15,23-25$,

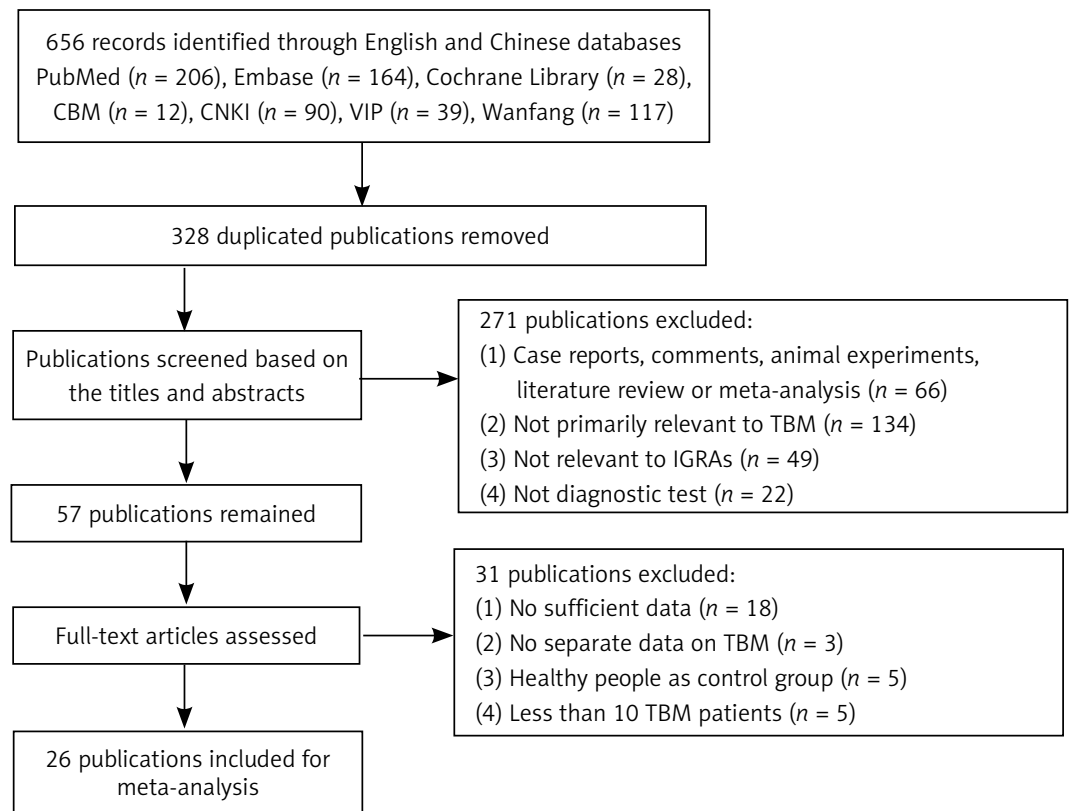

Figure 1. Flow chart for studies identified and included in the present meta-analysis 
Table I. Principal characteristics of included studies

\begin{tabular}{|c|c|c|c|c|c|c|c|c|c|c|}
\hline \multirow[t]{2}{*}{ Author } & \multirow[t]{2}{*}{ Year } & \multirow[t]{2}{*}{ Country } & \multirow[t]{2}{*}{ Study design } & \multirow{2}{*}{$\begin{array}{c}\text { TBM } \\
\text { patients }\end{array}$} & \multirow{2}{*}{$\begin{array}{c}\text { IGRA } \\
\text { method }\end{array}$} & \multirow[t]{2}{*}{ Sample } & \multicolumn{4}{|c|}{ Test result } \\
\hline & & & & & & & TP & FP & FN & TN \\
\hline \multirow[t]{2}{*}{ Zhang } & 2013 & China & Retrospective & 30 & T-SPOT.TB & PB & 23 & 4 & 7 & 26 \\
\hline & & & & & & CSF & 28 & 1 & 2 & 29 \\
\hline \multirow[t]{2}{*}{ Ling } & 2015 & China & Prospective & 12 & T-SPOT.TB & PB & 10 & 5 & 2 & 23 \\
\hline & & & & & & CSF & 11 & 2 & 1 & 26 \\
\hline \multirow[t]{2}{*}{ Thomas } & 2008 & India & Prospective & 11 & T-SPOT.TB & PB & 9 & 2 & 2 & 6 \\
\hline & & & & & & CSF & 9 & 0 & 1 & 7 \\
\hline \multirow[t]{2}{*}{ Park } & 2016 & Korea & Prospective & 49 & T-SPOT.TB & PB & 38 & 66 & 11 & 115 \\
\hline & & & & & & CSF & 28 & 16 & 12 & 117 \\
\hline \multirow[t]{2}{*}{ Kim } & 2010 & Korea & Prospective & 31 & T-SPOT.TB & PB & 22 & 20 & 9 & 30 \\
\hline & & & & & & CSF & 13 & 1 & 5 & 25 \\
\hline \multirow[t]{2}{*}{ Lu } & 2016 & China & Prospective & 30 & T-SPOT.TB & PB & 21 & 5 & 9 & 34 \\
\hline & & & & & QFT-GIT & CSF & 25 & 5 & 5 & 34 \\
\hline \multirow[t]{2}{*}{ Pan } & 2017 & China & Prospective & 53 & T-SPOT.TB & PB & 48 & 9 & 5 & 28 \\
\hline & & & & & & CSF & 32 & 1 & 21 & 36 \\
\hline \multirow[t]{2}{*}{ Pan } & 2015 & China & Prospective & 26 & T-SPOT.TB & PB & 26 & 10 & 0 & 7 \\
\hline & & & & & & CSF & 24 & 1 & 2 & 16 \\
\hline \multirow[t]{2}{*}{ Lu } & 2016 & China & Prospective & 20 & T-SPOT.TB & PB & 16 & 0 & 4 & 28 \\
\hline & & & & & & CSF & 19 & 1 & 1 & 27 \\
\hline Feng & 2009 & China & Prospective & 15 & T-SPOT.TB & PB & 12 & 0 & 3 & 11 \\
\hline Han & 2008 & China & Prospective & 13 & T-SPOT.TB & PB & 10 & 0 & 3 & 4 \\
\hline Cho & 2011 & Korea & Prospective & 35 & T-SPOT.TB & PB & 26 & 47 & 9 & 40 \\
\hline Zhang & 2017 & China & Prospective & 62 & T-SPOT.TB & PB & 56 & 7 & 6 & 53 \\
\hline Wang & 2017 & China & Prospective & 35 & T-SPOT.TB & PB & 26 & 3 & 9 & 27 \\
\hline Duan & 2017 & China & Prospective & 45 & T-SPOT.TB & PB & 41 & 6 & 4 & 44 \\
\hline Zheng & 2015 & China & Prospective & 12 & T-SPOT.TB & PB & 12 & 2 & 0 & 6 \\
\hline Jiang & 2016 & China & Prospective & 58 & T-SPOT.TB & PB & 50 & 2 & 8 & 15 \\
\hline Wang & 2016 & China & Retrospective & 54 & T-SPOT.TB & PB & 45 & 10 & 9 & 24 \\
\hline Cheng & 2017 & China & Retrospective & 61 & T-SPOT.TB & PB & 49 & 3 & 12 & 29 \\
\hline Wang & 2014 & China & Prospective & 26 & T-SPOT.TB & CSF & 24 & 3 & 2 & 21 \\
\hline Quan & 2008 & China & Retrospective & 25 & T-SPOT.TB & CSF & 21 & 4 & 4 & 23 \\
\hline Patel & 2010 & Africa & Prospective & 38 & T-SPOT.TB & CSF & 32 & 13 & 6 & 35 \\
\hline Chen & 2015 & China & Retrospective & 52 & QFT-GIT & PB & 42 & 5 & 10 & 42 \\
\hline $\mathrm{Mu}$ & 2015 & China & Prospective & 32 & QFT-GIT & PB & 28 & 2 & 4 & 28 \\
\hline Qian & 2012 & China & Retrospective & 32 & QFT-GIT & PB & 28 & 5 & 4 & 51 \\
\hline Vidhate & 2011 & India & Retrospective & 36 & QFT-GIT & PB & 16 & 6 & 20 & 10 \\
\hline
\end{tabular}

CSF - cerebrospinal fluid, PB - peripheral blood, TP - true positive, FP - false positive, FN - false negative, TN - true negative, IGRA method - interferon- $\gamma$ - release assay method.

27, 32], whereas other publications did not report on blinding. Microbiological confirmation was used as the only reference standard to diagnose TBM in one publication [36], while in the remaining publications, clinical standards for TBM diagnosis were used as an alternative. A number of publications performed IGRAs on two types of specimens. As a consequence, 35 unique studies were defined from 26 articles. Peripheral blood (PB) IGRAs were used in 23 studies [15, 22-39, 42-45], while CSF IGRAs were used in 12 studies [14, 15, 22-29, 40, 41].

\section{Quality assessment}

Figure 2 shows the quality of the studies included in the meta-analysis. Overall, the risk of bias was low across four domains. Index test and reference standard domain were judged to be at unclear risk 
of bias in the studies not reporting on the employment of a blinding method. Reference standard domain showed high concerns of applicability due to the limitations of traditional pathogenic inspection and clinical standards. Additional information about the quality of included studies is shown in Figure 2.

\section{Overall meta-analysis of IGRAs}

The pooled estimates are shown in Figure 3. For PB IGRAs: the sensitivities varied from 0.44 to 0.91 ; the specificity varied from 0.41 to 1.00 . Pooled estimates for PLR, NLR, and DOR were 4.23 (95\% Cl: 2.95-6.07), 0.24 (95\% Cl: 0.19-0.32), and 21.06 (11.91-37.24), respectively. There was moderate heterogeneity in sensitivity and high heterogeneity in specificity between studies.

As for CSF IGRAs: the sensitivities varied from 0.60 to 0.95 ; the specificity varied from 0.73 to 1.00. Pooled estimates for PLR, NLR, and DOR were 7.87 (95\% Cl: 4.98-12.46), 0.19 (95\% Cl: 0.13-0.29) and 47.74 (25.02-91.12), respectively. $I^{2}$ values of these parameters indicated moderate heterogeneity among studies. When comparing overall diagnostic accuracy of PB IGRAs with CSF IGRAs, the latter showed significantly higher specificity and PLR $(p<0.05)$ than the former.

\section{Heterogeneity analysis}

The Spearman rank correlation test indicated no threshold effect in the PB IGRAs (coefficient = -0.056, $p=0.801$ ) and CSF IGRAs studies (coefficient $=0.036, p=0.939$ ). The summary of subgroup analyses is shown in Table II.

On subgroup analyses of PB IGRAs, the studies reporting a blinding method were associated with lesser heterogeneity in sensitivity and specificity. Correspondingly, on subgroup analyses of CSF IGRAs, studies with sample size $<30$ and those in which a blinding method were not reported were related to obvious decreased heterogeneity in sensitivity and specificity. Table III assesses the potential factors associated with IGRAs' diagnostic accuracy by using multivariate meta-regression. However, none of the factors significantly influenced the relative DOR of IGRAs for the diagnosis of TBM.

\section{Publication bias}

Egger's test was performed to assess the publication bias of included studies. There was a risk of publication bias in the meta-analysis of CSF IGRAs $(p<0.05)$. No evidence of publication bias was found in PB IGRAs $(p>0.05)$.

\section{Comparison on diagnostic performance among ADA, new techniques and IGRAs for TBM}

Studies aimed at analyzing the test accuracy in diagnosing TBM were reviewed. The diagnos-

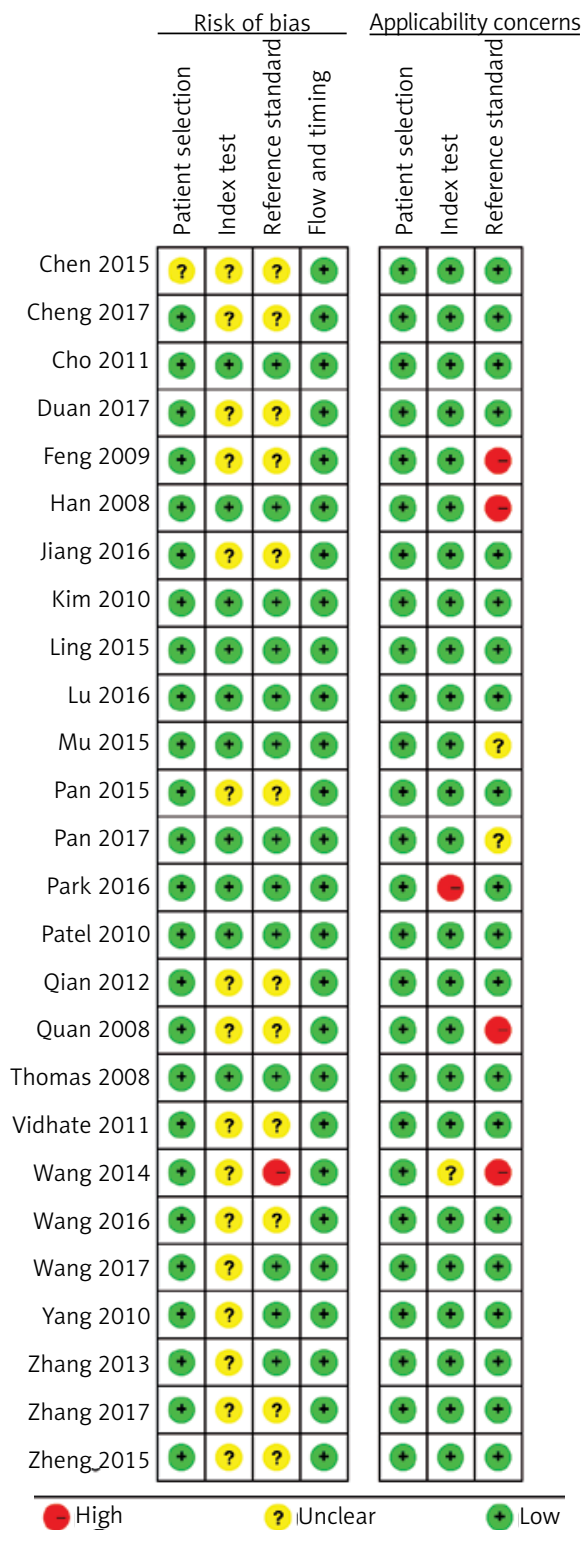

Figure 2. Methodological quality evaluation results of 26 publications using the QUADAS-2 tool

tic performance of ADA was extracted from a meta-analysis [46]. Applications in diagnosing TBM of four new techniques were also collected. As shown in Table IV, the sensitivity of the IGRA was slightly lower than ADA and the other four techniques while its specificity was much lower.

\section{Discussion}

The results of the present meta-analysis indicated that PB and CSF IGRAs could neither diagnose nor rule out TBM. Thus, in agreement with the previous meta-analysis [16], the diagnostic performance of IGRAs for TBM is suboptimal. Several reasons may explain this. First, PB IGRAs reflect systematic condition of the patients, which means that we may get false positive test results in nonTBM patients with tuberculosis at other sites than 
A
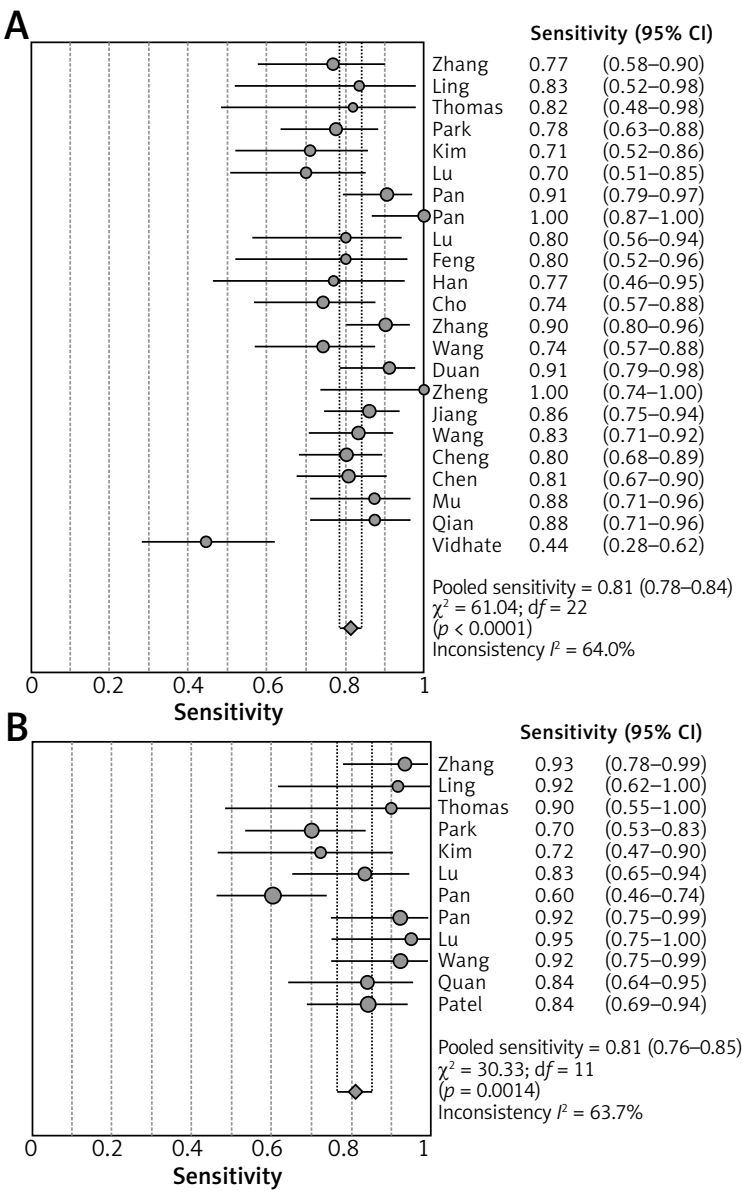

Figure 3. Forest plots of sensitivity and specificity for PB IGRAs (A) and CSF IGRAs (B)
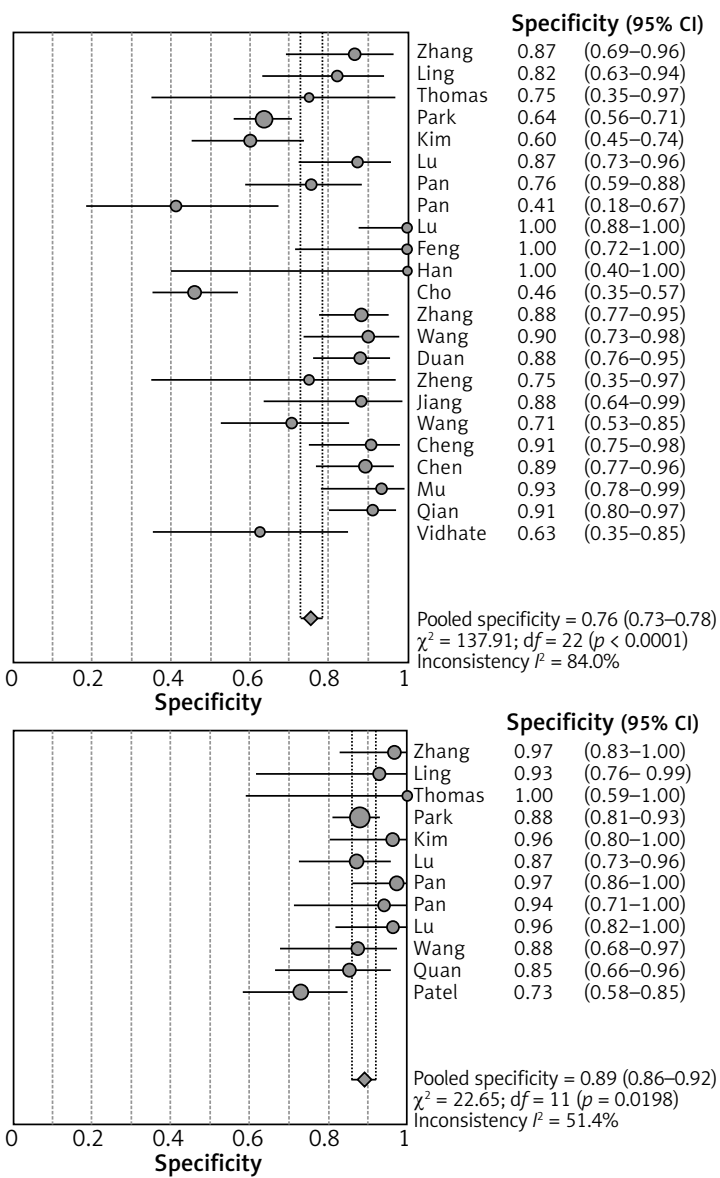

the brain. In addition, false positives owing to latent tuberculosis infection (LTBI) in non-TBM patients may also contribute to compromised specificity, which is one of the reasons why the World Health Organization recommends against the use of IGRAs as diagnostic markers for active tuberculosis in countries with high background LTBI rates [47]. In the present meta-analysis, the use of IGRAs on the CSF specimens displayed significant higher specificity than on the PB, which supports the theory that more $M$. Tb-specific lymphocytes are compartmentalized at the infected sites [48]. However, indeterminate results are common in CSF IGRAs. Relatively large CSF volumes $(5-10 \mathrm{ml})$ that are necessary to provide sufficient cells for the detection are not always possible to achieve in clinical practice. These two factors impaired the diagnostic value of CSF IGRAs.

As there are many limitations in microbiological tests, new approaches were developed for detection of $M$. Tb [49-52]. Compared with ZiehlNeelsen staining and culture, IGRAs break new ground in TBM diagnosis as they are much more rapid and simple. However, in comparison to CSF ADA, IGRAs did not show remarkable superiority in terms of cost, turn-around time and diagnostic accuracy. Some new techniques for TBM diagnosis listed in Table IV showed obvious advantages in detection time, test procedures, accessibility and cost. Meanwhile, they showed promising diagnostic value for TBM. However, to date, few studies with large sample size have been conducted to investigate the role of these novel techniques in detection of TBM. More data are needed to validate the value of these new techniques in practical application in TBM diagnosis.

Currently, there are many guidelines on IGRAs for tuberculosis infection from different countries and supranational organizations but none for TBM [53]. According to the guidelines on IGRAs for tuberculosis combined with the results of this meta-analysis, some recommendations on IGRAs for TBM were summarized below. Clearly, IGRAs could not replace the microbiological tests. Given the significantly higher costs and disproportionate diagnostic performance, IGRAs were even inferior to some traditional tests such as CSF ADA in resource-limited and high TB burden settings. Even so, IGRAs could provide supplementary information in certain clinical situations, e.g. in immunocompromised patients where IGRAs are less affected by immunosuppression; highly suspected TBM patients but negative on microscopy and culture; or in the differential diagnosis of infection 
Table II. Subgroup analysis for exploration of factors influencing heterogeneity

\begin{tabular}{|c|c|c|c|c|c|c|}
\hline Subgroup & $\begin{array}{l}\text { Number } \\
\text { of studies }\end{array}$ & $\begin{array}{c}\text { Sensitivity } \\
(95 \% \mathrm{Cl})\end{array}$ & $I^{2}(\%)$ & $\begin{array}{c}\text { Specificity } \\
(95 \% \mathrm{Cl})\end{array}$ & $I^{2}(\%)$ & $\begin{array}{c}\text { DOR } \\
(95 \% \mathrm{Cl})\end{array}$ \\
\hline \multicolumn{7}{|l|}{ PB IGRAs: } \\
\hline \multicolumn{7}{|l|}{ TB burden: } \\
\hline High & 20 & $0.82(0.79-0.85)$ & 66.7 & $0.85(0.82-0.88)$ & 63.1 & $29.18(17.45-48.97)$ \\
\hline Low & - & - & - & - & - & - \\
\hline \multicolumn{7}{|l|}{ Blind: } \\
\hline Yes & 6 & $0.80(0.74-0.86)$ & 26.4 & $0.62(0.57-0.67)$ & 73 & $7.46(3.36-16.57)$ \\
\hline NR & 17 & $0.82(0.78-0.85)$ & 70.4 & $0.86(0.83-0.89)$ & 66.6 & $31.05(16.98-56.76)$ \\
\hline \multicolumn{7}{|l|}{ Sample size: } \\
\hline$<30$ & 7 & $0.87(0.79-0.93)$ & 54.8 & $0.82(0.73-0.89)$ & 81.0 & $35.40(13.31-94.17)$ \\
\hline$\geq 30$ & 16 & $0.80(0.77-0.83)$ & 66.5 & $0.75(0.72-0.78)$ & 85.6 & $18.33(9.48-35.44)$ \\
\hline \multicolumn{7}{|l|}{ Method: } \\
\hline TSPOT.TB & 19 & $0.83(0.80-0.86)$ & 47.1 & $0.73(0.70-0.76)$ & 84.1 & $20.27(11.36-36.15)$ \\
\hline QFT-GIT & 4 & $0.75(0.67-0.82)$ & 86.6 & $0.88(0.82-0.93)$ & 64.3 & $22.94(3.07-171.69)$ \\
\hline \multicolumn{7}{|c|}{ Reference standard: } \\
\hline Clinical & 22 & $0.81(0.78-0.84)$ & 62.5 & $0.76(0.73-0.78)$ & 84.8 & $20.56(11.52-36.67)$ \\
\hline Microbiological & - & - & - & - & - & - \\
\hline \multicolumn{7}{|l|}{ CSF IGRAs: } \\
\hline \multicolumn{7}{|l|}{ TB burden: } \\
\hline High & 10 & $0.83(0.78-0.88)$ & 65 & $0.89(0.85-0.92)$ & 56.6 & $59.28(28.14-124.89)$ \\
\hline Low & - & - & - & - & - & - \\
\hline \multicolumn{7}{|l|}{ Blind: } \\
\hline Yes & 6 & $0.73(0.66-0.80)$ & 55.7 & $0.88(0.84-0.92)$ & 70.4 & $24.77(12.84-47.79)$ \\
\hline NR & 6 & $0.90(0.84-0.94)$ & 0.0 & $0.91(0.85-0.95)$ & 0.0 & $80.44(31.87-203.03)$ \\
\hline \multicolumn{7}{|l|}{ Sample size: } \\
\hline$<30$ & 6 & $0.91(0.84-0.95)$ & 0.0 & $0.92(0.85-0.96)$ & 0.0 & $84.33(34.58-205.62)$ \\
\hline$\geq 30$ & 6 & $0.76(0.69-0.81)$ & 68.9 & $0.88(0.84-0.92)$ & 71.1 & $31.07(14.30-67.50)$ \\
\hline
\end{tabular}

DOR - diagnostic odds ratio, $N R$ - not reported.

Table III. Multivariate meta-regression to evaluate factors associated with interferon- $\gamma$ release assay accuracy in tuberculous meningitis

\begin{tabular}{|lccc|}
\hline Covariate & Coefficient & $P$-value & RDOR (95\% Cl) \\
\hline PB IGRAs: & & & \\
\hline TB burden & -0.525 & 0.5679 & $0.59(0.09-3.98)$ \\
\hline Blind & 1.581 & 0.1371 & $4.86(0.57-41.34)$ \\
\hline Sample size & -0.341 & 0.6782 & $0.71(0.13-3.93)$ \\
\hline Method & 0.474 & 0.4974 & $1.61(0.38-6.84)$ \\
\hline Reference standard & -0.458 & 0.8327 & $0.63(0.01-58.41)$ \\
\hline CSF IGRAs: & & & $1.95(0.39-9.76)$ \\
\hline TB burden & 0.666 & 0.3616 & $0.54(0.12-2.46)$ \\
\hline Blind & -0.614 & 0.3699 & $0.42(0.09-1.95)$ \\
\hline Sample size & -0.868 & 0.2227 & \\
\hline
\end{tabular}

$R D O R$ - relative diagnostic odds ratio.

with NTM. Notably, compared with resource-limited and high-incidence settings, IGRAs played a more important role in their additive value in high-income and low-incidence countries.
Apart from the above, findings from this metaanalysis revealed no significant difference in the DOR of TSPOT.TB and QFT-GIT, which may be due to insufficient studies of QFT-GIT for TBM. In 
Table IV. Diagnostic performance of ADA, nRT-PCR, OTNPCR-LFST, LAMP and IGRAs for TBM

\begin{tabular}{|lcccccc|}
\hline Test method & Year & $\begin{array}{c}\text { Study } \\
\text { number }\end{array}$ & $\begin{array}{c}\text { TBM } \\
\text { patient }\end{array}$ & Sample & Sensitivity (95\% Cl) & Specificity (95\% Cl) \\
\hline ADA [46] & 2017 & 20 & 741 & CSF & $0.89(0.84-0.92)$ & $0.91(0.87-0.93)$ \\
\hline nRT-PCR [49] & 2017 & 1 & 14 & CSF & $0.86(0.60-0.96)$ & $1.00(0.95-1.00)$ \\
\hline OTNPCR-LFST [50] & 2017 & 1 & 91 & CSF & $0.89(0.82-0.95)$ & $1.00(0.95-1.00)$ \\
\hline IS6110 LAMP [51] & 2016 & 1 & 150 & CSF & $0.83(0.76-0.88)$ & $1.00(0.96-1.00)$ \\
\hline MPB64 LAMP [51] & 2016 & 1 & 150 & CSF & $0.87(0.80-0.92)$ & $1.00(0.96-1.00)$ \\
\hline PB IGRAs & Present & 26 & 893 & PB & $0.81(0.78-0.84)$ & $0.76(0.73-0.78)$ \\
\hline CSF IGRAs & Present & 26 & 893 & CSF & $0.81(0.76-0.85)$ & $0.89(0.86-0.92)$ \\
\hline
\end{tabular}

$A D A$ - adenosine deaminase assay, $n R T$-PCR - nested real-time polymerase chain reaction, OTNPCR-LFST - one-tube nested PCR-lateral flow strip test, LAMP - loop-mediated isothermal amplification.

clinical practice, both of the methods have their strengths and weakness. The probability of laboratory error is higher for the TSPOT.TB on account of more complicated technical demand and processing steps than QFT-GIT. On the other hand, TSPOT. TB was deemed to be more stable and sensitive for diagnosing tuberculosis. But in fact, there are few reports comparing the performance of QFTGIT and TSPOT.TB assays directly in patients with clinically suspected TBM. Thus, larger and parallel studies are required to compare the performance between the two methods for diagnosing TBM.

This study has several strengths. First, the search strategy was broader than the previous one as we incorporated many databases and no language restriction to minimize the number of missing publications. Nineteen additional studies were identified for review, including two publications ignored by the previous meta-analysis. Second, included studies met the predefined inclusion plan, reducing the bias produced by inclusion of patients to some extent. Third, this meta-analysis comprehensively evaluated the diagnostic value of TSPOT.TB and QFT-GIT, two types of IGRAs, as well as two types of samples (PB and CSF). Above all, the large sample size was the major strength of this meta-analysis.

This meta-analysis has some limitations. First, there were considerable heterogeneities of the selected studies, which may have led to overestimation of the pooled estimates. As the diagnostic criteria, cut-off value, disease prevalence and other populations characteristics varied among studies, the heterogeneity is to be expected. The factors included in the meta-regression analysis failed to explain the heterogeneity. Reference standards were divided into two groups in meta-regression: clinical and microbiological. As there is no unified criterion for TBM diagnosis, this factor is too mixed and difficult to make further subdivisions, which may be partly responsible for the inexplicable heterogeneity. Second, the absolute and most widely used diagnostic tools for TBM are smear micros- copy and culture yet are negative in a significant proportion of TBM cases, leading researchers to use alternate clinical reference standards. In view of this, we did not restrict diagnostic criteria to microbiological confirmation, which is commonly not achievable in a routine clinical setting. This may produce bias. In order to minimize the occurrence of misdiagnosis of TBM, only studies with predefined and rigorous diagnostic criteria were included in the current analysis. Third, several studies did not report on blinding. And there was potential publication bias in the meta-analysis for CSF IGRAs.

In conclusion, since IGRAs suffer from problems such as high cost, rigorous technical requirements, existence of indeterminate results and suboptimal diagnostic value, these assays are unsuitable for use as biomarkers for standalone TBM diagnosis.

\section{Acknowledgments}

Thanks for the effort made by all authors of studies included in this meta-analysis.

\section{Conflict of interest}

The authors declare no conflict of interest.

\section{References}

1. WHO. Global tuberculosis control 2017. World Health Organization, Geneva 2017.

2. Thwaites GE, Hien TT. Tuberculous meningitis: many questions, too few answers. J Lancet Neurol 2013; 12: 999-1010.

3. Porkert MT, Sotir M, Parrott-Moore P, Blumberg HM. Tuberculous meningitis at a large inner-city medical center. Am J Med Sci 1997; 313: 325-31.

4. Jiang YG, Chen J, Peng Y. Tuberculous brainstem abscess: case report. Neurosurgery 2009; 65: E1206-7.

5. Stewart SM. The bacteriological diagnosis of tuberculous meningitis. J Clin Pathol 1953; 6: 241-2.

6. Peng J, Zhang HY, Wu LW, et al. Diagnosis of disease-specific proteins in cerebrospinal fluid of children infected with tuberculous meningitis. Neural Regen Res 2010; 5: 927-32. 
7. Arend SM, van Meijgaarden KE, de Boer K, et al. Tuberculin skin testing and in vitro T cell responses to ESAT-6 and culture filtrate protein 10 after infection with Mycobacterium marinum or M. kansasii. J Infect Dis 2002; 186: 1797-807.

8. Pai M, Flores LL, Pai N, Hubbard A, Riley LW, Colford JM Jr. Diagnostic accuracy of nucleic acid amplification tests for tuberculosis meningitis: a systematic review and meta-analysis. Lancet Infect Dis 2003; 3: 633-43.

9. Cattamanchi A, Smith R, Steingart KR, et al. Interferon-gamma release assays for the diagnosis of latent tuberculosis infection in HIV-infected individuals: a systematic review and meta-analysis. J Acquir Immune Defic Syndr 2011; 56: 230-8.

10. Chen J, Zhang R, Wang J, Liu L, Zheng Y, Shen Y, Qi T, $\mathrm{Lu} \mathrm{H}$. Interferon-gamma release assays for the diagnosis of active tuberculosis in HIV-infected patients: a systematic review and meta-analysis. PLoS One 2011; 6: e26827.

11. Machingaidze S, Wiysonge CS, Gonzalez-Angulo Y, et al. The utility of an interferon gamma release assay for diagnosis of latent tuberculosis infection and disease in children: a systematic review and meta-analysis. Pediatr Infect Dis J 2011; 30: 694-700.

12. Metcalfe JZ, Everett CK, Steingart KR, et al. Interferon-gamma release assays for active pulmonary tuberculosis diagnosis in adults in low- and middle-income countries: systematic review and meta-analysis. J Infect Dis 2011; 204 (Suppl 4): S1120-9.

13. Xie X, Chen JW, Li F, Tian J, Gao JS, Zhang D. A T-cellbased enzyme-linked immunospot assay for tuberculosis screening in Chinese patients with rheumatic diseases receiving infliximab therapy. Clin Exp Med 2011; 11: 155-61.

14. Patel VB, Singh R, Connolly C, et al. Cerebrospinal T-cell responses aid in the diagnosis of tuberculous meningitis in a human immunodeficiency virus- and tuberculosis-endemic population. Am J Respir Crit Care Med 2010; 182: 569-77.

15. Kim SH, Cho OH, Park SJ, et al. Rapid diagnosis of tuberculous meningitis by $\mathrm{T}$ cell-based assays on peripheral blood and cerebrospinal fluid mononuclear cells. J Clin Infect Dis 2010; 50: 1349-58.

16. Yu J, Wang ZJ, Chen LH, Li HH. Diagnostic accuracy of interferon-gamma release assays for tuberculous meningitis: a meta-analysis. Int J Tuberc Lung Dis 2016; 20 494-9.

17. Devillé WL, Buntinx F, Bouter LM, et al. Conducting systematic reviews of diagnostic studies: didactic guidelines. BMC Med Res Methodol 2002; 2: 9.

18. Deeks JJ. Systematic reviews in health care: systematic reviews of evaluations of diagnostic and screening tests. BMJ 2001; 323: 157-62.

19. Wei C, Wen-En L, Yang-Ming L, Shan L, Yi-Ming Z. Diagnostic accuracy of loop-mediated isothermal amplification in detection of Clostridium difficile in stool samples: a meta-analysis. Arch Med Sci 2015; 11: 927-36.

20. Chen A, Chen X, Shen Y, Li W. Modalities of ventricular pacing for cardiac resynchronization therapy in patients with heart failure: a meta-analysis and systematic review. Arch Med Sci 2017; 13: 1006-17.

21. Egger M, Davey Smith G, Schneider M, Minder C. Bias in meta-analysis detected by a simple, graphical test. BMJ 1997; 315: 629-34.

22. Zhang X, Wu R, Fan X. Study on the early diagnostic value of T-SPOT.TB assay detecting mononuclear cells in cerebrospinal fluid of patients with tuberculous meningitis. Chin J Contemp Neurol Neurosurg 2013; 13: 110-5.

23. Ling Q, Zhang L, Zhang Y, Shi X, Zhang Y, Liu X. Diagnostic value of T-cell interferon-gamma release assays on cerebrospinal fluid for tuberculous meningitis. PLoS One 2015; 10: e0141814.

24. Thomas MM, Hinks TS, Raghuraman S, et al. Rapid diagnosis of $M$. tuberculosis meningitis by enumeration of cerebrospinal fluid antigen-specific T cells. Int J Tuberc Lung Dis 2008; 12: 651-7.

25. Park KH, Lee MS, Kim SM, et al. Diagnostic usefulness of T-cell based assays for tuberculous meningitis in HIV-uninfected patients. J Infect 2016; 72: 486-97.

26. Lu D, Chen C, Yu S, Chen S. Diagnosis of tuberculous meningitis using a combination of peripheral blood T-SPOT.TB and cerebrospinal fluid interferon-gamma detection methods. J Lab Med 2016; 47: 6-12.

27. Pan LP, Liu F, Zhang JL, et al. Interferon-gamma release assay performance of cerebrospinal fluid and peripheral blood in Tuberculous meningitis in China. Biomed Res Int 2017; 2017: 8198505.

28. Pan YY, Li J, Wang XJ, Cheng XX. Application of ELISPOT in the diagnosis of tuberculous meningitis. J Clin Pulmonary Med 2015; 20: 204-6.

29. Lu JH, Wu YZ, Yang Y, et al. Application value of T cell enzyme-linked immunospot assay in the diagnosis of tuberculosis meningitis. Chin J Health Lab Tec 2016; 26: 816-8.

30. Feng Y, Shao LY, Meng CY, et al. TSPOT.TB in the diagnosis of tuberculous meningitis. J Infect Dis Info 2009; 22: 301-4.

31. Han W, Zhang W, He P, et al. Analysis of the utility of enzyme-linked immunospot assay in children with tuberculous meningitis. J Xinjiang Med Univ 2008; 31: 617-22.

32. Cho OH, Park KH, Kim SM, et al. Diagnostic performance of T-SPOT.TB for extrapulmonary tuberculosis according to the site of infection. J Infect 2011; 63: 362-9.

33. Zhang CY, Chi F, Zhang X, et al. Comparison of the different methods in the diagnosis of tuberculous meningitis. Chin J Compl Cas 2011; 16: 356-63.

34. Wang JJ, Ma L. Application value of different testing methods in the diagnosis of tuberculous meningitis. J China Medical Herald 2017; 14: 119-22.

35. Duan SX. Research of detection of tuberculosis infected $T$ cells on diagnostic value in tuberculous meningitis. J Med Forum 2017; 38: 61-2.

36. Zheng QJ, Xu FP, Lin ZR, et al. Effects of T-SPOT on early differential diagnosis of cryptococcal meningitis and tubercular meningitis. J Fujian Med 2015; 37: 114-6.

37. Jiang Z, Wu WH, Xu F, et al. Effect analysis of using tuberculosis infection $T$ cells detection in the clinical diagnosis of tuberculous meningitis. Chin J Nosocomiol 2016; 26: 3376-73.

38. Wang ZC, Bai GG, Wang XH, et al. Tuberculous meningitis clinical laboratory evaluation of new developments. J Lab Med Clin 2016; 13: 119-21.

39. Cheng HY, Wang DP, Bao XD, et al. Diagnostic value of blood T-SPOT in the diagnosis of tuberculous meningitis. Chin J Clin 2017; 11: 357-60.

40. Wang Y, Zhou H, Liu L, et al. Clinical study of the setection of T-SPOT.TB in Diagnosis of tuberculosis meningitis. Chin J Health Stand Manag 2014; 5: 68-7.

41. Quan C, Qiao J, Xiao B, et al. A comparative evaluation of enzyme linked immunospot assay and IS6110 polymerase chain reaction for early diagnosis of tuberculosis meningitis. Chin J Neurol 2008; 41: 176-9. 
42. Chen Q, Xu T. QuantifERON - TB gold in-tube assay for diagnosis of tuberculous meningitis in children. Chin J Clin Infect Dis 2015; 8: 161-3.

43. Mu F, He H, Tan T, Li Y. The value of interferon gamma release assays in the diagnosis of tuberculous meningitis in children. J Clin Pediatr 2015; 33: 242-6.

44. Qian F, He J, Shen Yi. Application of interferon-gamma release assay in diagnosis of tuberculous meningitis. Chin J Health Labor Technol 2012; 22: 1037-41.

45. Vidhate MR, Singh MK, Garg RK, et al. Diagnostic and prognostic value of Mycobacterium tuberculosis complex specific interferon-gamma release assay in patients with tuberculous meningitis. J Infect 2011; 62: 400-3.

46. Pormohammad A, Riahi SM, Nasiri MJ, et al. Diagnostic test accuracy of adenosine deaminase for tuberculous meningitis: a systematic review and meta-analysis. J Infect 2017; 74: 545-54.

47. World Health Organization. 2011. Use of tuberculosis interferon-gamma release assays (IGRAs) in low- and middle-income countries: policy statement. World Health Organization, Geneva, Switzerland 2011.

48. Breen RA, Barry SM, Smith CJ, et al. The clinical application of a rapid lung-orientated TB immunoassay in individuals with possible tuberculosis. J Thorax 2008; 63: 67-71.

49. Gualberto FAS, Gonçalves MG, Fukasawa LO, et al. Performance of nested RT-PCR on CSF for tuberculous meningitis diagnosis in HIV-infected patients. Int J Tuberc Lung Dis 2017; 21: 1139-44.

50. Sun Y, Chen J, Li J, et al. Novel approach based on onetube nested PCR and a lateral flow strip for highly sensitive diagnosis of tuberculous meningitis. PLoS One 2017; 12: e0186985.

51. Modi M, Sharma K, Sharma M, et al. Multitargeted loop-mediated isothermal amplification for rapid diagnosis of tuberculous meningitis. Int J Tuberc Lung Dis 2016; 20: 625-30.

52. Peng J, Liu WE, Li HL, et al. Evaluation of our self-designed nanometer silicon membrane sandwich cup system for diagnosing tuberculosis. Clin Respir J 2016; 10: 647-52.

53. Denkinger CM, Dheda K, Pai M. Guidelines on interferon-gamma release assays for tuberculosis infection: concordance, discordance or confusion? Clin Microbiol Infect 2011; 17: 806-14. 